\title{
BRAF-activated WT1 contributes to cancer growth and regulates autophagy and apoptosis in papillary thyroid carcinoma
}

Xing Chen ${ }^{1,2}$, Shan Lin ${ }^{2,3}$, Ying Lin ${ }^{4}$, Songsong Wu ${ }^{5}$, Minling Zhuo ${ }^{6}$, Ailong Zhang ${ }^{1,2}$, Junjie Zheng ${ }^{1,2}$ and Zhenhui You ${ }^{1,2^{*}}$

\begin{abstract}
Background: Papillary thyroid carcinoma (PTC) is one of most prevalent malignant endocrine neoplasms, and it is associated with a high frequency of BRAF gene mutations, which lead to lymphatic metastasis and distant metastasis that promote tumor progression. The molecular mechanism of PTC and the role of BRAF mutation in PTC progression and development need to be further elucidated.
\end{abstract}

Methods: In this study, a comprehensive bioinformatics analysis was performed to identify the differentially expressed genes and signaling pathways in thyroid cancer patients carrying mutant BRAF. Then, we confirmed the prognostic role of WT1 in thyroid cancer patients. Immunohistochemistry was performed to measure the expression profile of WT1 in PTC tissue. Lentivirus shWT1 was transfected into BRAF ${ }^{\mathrm{V} 600 \mathrm{E}}$ (mutant) PTC cells to stably inhibit WT1 expression. CCK-8, EdU, immunofluorescence, colony formation, cell migration, cell wound healing, apoptosis and autophagy assays were performed to assess the biological functions of WT1 in BRAFV00E PTC cells. RNA sequencing, immunohistochemistry and immunoblotting were performed to explore the molecular mechanism of WT1 in BRAF ${ }^{\mathrm{V} 600 \mathrm{E}}$ PTC cells.

Results: The results confirmed that "epithelial cell proliferation", "apoptosis" and "selective autophagy" were closely associated with this BRAF mutant in these thyroid cancer patients. Knocking down BRAF-activated WT1 effectively inhibited the proliferation and migration of BRAF ${ }^{\mathrm{V} 600 E}$ PTC cells. Silencing WT1 significantly inhibited autophagy and promoted the apoptosis of BRAF ${ }^{\mathrm{V} 600 \mathrm{E}}$ PTC cells. Mechanistic investigations showed that silencing WT1 expression remarkably suppressed the AKT/mTOR and ERK/P65 signaling pathways in BRAFV600E PTC cells.

Conclusion: All these results indicate that WT1 is a promising prognostic biomarker and facilitates PTC progression and development of cells carrying the BRAF ${ }^{\mathrm{V} 600 \mathrm{E}}$ mutation.

Keywords: PTC, WT1, Autophagy, Apoptosis, BRAF ${ }^{\mathrm{V} 600 \mathrm{E}}$

\footnotetext{
*Correspondence: youzh21@sina.com

${ }^{1}$ Department of General Surgery, Fujian Medical University Provincial

Clinical Medical College, Fujian Provincial Hospital, Fuzhou 350001, Fujian, China

Full list of author information is available at the end of the article
}

\section{Background}

Thyroid cancer is one of the most prevalent malignant endocrine neoplasms, with approximately $5 \%$ of newly diagnosed cases, with the morbidity and mortality gradually increasing [1]. Papillary thyroid cancer (PTC) accounts for approximately $85 \%$ of all thyroid cancers and is the main thyroid malignancy subtype [2]. Despite improvements in current treatment methods, including original author(s) and the source, provide a link to the Creative Commons licence, and indicate if changes were made. The images or other third party material in this article are included in the article's Creative Commons licence, unless indicated otherwise in a credit line to the material. If material is not included in the article's Creative Commons licence and your intended use is not permitted by statutory regulation or exceeds the permitted use, you will need to obtain permission directly from the copyright holder. To view a copy of this licence, visit http://creativecommons.org/licenses/by/4.0/. The Creative Commons Public Domain Dedication waiver (http://creativeco mmons.org/publicdomain/zero/1.0/) applies to the data made available in this article, unless otherwise stated in a credit line to the data. 
surgical management, radioiodine therapy, and levothyroxine treatment, some patients experience metastatic and aggressive spread of this cancer, leading to poor clinical outcomes [3]. Therefore, it is necessary to explore the molecular mechanism of PTC progression and search for valuable biomarkers and therapeutic targets to conquer this disease.

In recent years, with the development of high-throughput detection techniques, it has become more convenient to identify promising biomarkers to predict the prognosis and recurrence of various malignancies [4-6]. Based on these techniques, the measurement of gene mutation status, including BRAF, RAS and TERT mutations, also serves as an effective method for exploring regulatory mechanisms and provides a strategy for the individualized treatment of PTC in patients [7-9]. BRAF mutation is the most common genetic lesion in PTC, accounting for $50 \%$ of all cases, and the $\mathrm{BRAF}^{\mathrm{V} 600 \mathrm{E}}$ mutation is the most frequent mutation (95\%) among all mutation types [10]. The $\mathrm{BRAF}^{\mathrm{V} 600 \mathrm{E}}$ mutant activates the MARK pathway and promotes cancer progression in PTC [11]. Targeting the BRAF ${ }^{\mathrm{V} 600 \mathrm{E}}$ mutant is a useful method for treating PTC. However, the underlying mechanism of BRAF function in PTC progression remains unknown.

Wilms' tumor 1 (WT1), located on chromosome $11 \mathrm{p} 13$, encodes a transcription factor that includes a proline-/glutamine-rich domain at the $\mathrm{N}$-terminus and four zinc finger motif DNA-binding domains in the C-terminus [12]. It has been reported that WT1 functions as both an oncogene and tumor suppressor in multiple malignancies, playing a critical role in cell survival, proliferation and differentiation [13-15]. The expression level of WT1 also is an independent prognostic factor for a variety of cancers, including breast cancer, colorectal cancer and gynecological cancer, because of its superior predictive performance [16-18]. However, the value of WT1 expression as a prognostic indicator in PTC remains unclear.

Autophagy is a catabolic process by which lysosomes degrade dysfunctional cellular components inside the cell. Three kinds of autophagy have been described to date: microautophagy, macroautophagy and chaperone-mediated autophagy [19]. The autophagy process is closely related to different diseases, such as diabetes, colitis, cardiovascular disorders and cancer [20]. Apoptosis is considered the most common programmed cell death in mammalian cells and is involved in physiological and pathological processes, including normal cell turnover, chemical-induced cell death and regulation of the immune system [21]. Growing evidence suggests that regulating autophagy and apoptosis is an effective cancer treatment because these processes play important roles in PTC progression and development. In a previous study, the expression levels of WT1 were positively correlated with active autophagy in human osteosarcoma cells [22]. WT1 in breast cancer cells has also been shown to contribute to an increased cell proliferation rate and a reduced apoptosis rate [23]. However, the role of WT1 in PTC progression and the underlying mechanism of WT1 in PTC need to be further explored.

In this study, 67 differentially expressed genes correlating with both survival outcome and BRAF mutant were eventually obtained through the analyses of the genes and signaling pathways between PTC patients containing BRAF mutant and BRAF wild-type. Considering these differentially expressed genes, we found that WT1 may be a valuable biomarker for PTC in patients. Then, we analyzed the effect of the WT1 expression level on the proliferation, migration and tumor growth of $\mathrm{BRAF}^{\mathrm{V} 600 \mathrm{E}}$ PTC cells. In addition, we explored the role of WT1 in regulating autophagy and apoptosis in $\mathrm{BRAF}^{\mathrm{V} 600 \mathrm{E}} \mathrm{PTC}$ cells and searched for the potential mechanism of WT1 action in BRAF ${ }^{\mathrm{V} 600 \mathrm{E}}$ PTC cells. This study explored the oncogenic role of WT1 in PTC progression and development, and the results suggested that WT1 may be a novel therapeutic target in PTC patients with BRAF mutation.

\section{Materials and methods Identification of differentially expressed genes (DEGs)}

Somatic mutation profiles, mRNA-sequence data and clinical information of thyroid carcinoma (THCA) patients were downloaded from the TCGA database (https://portal.gdc.cancer.gov/). The THCA cohort dataset included 58 nontumor samples and 510 THCA samples (containing 270 wild-type BRAF samples and 240 mutant BRAF samples). Then, we used the "edgeR" software package (version 3.6.3, https://www.r-project. org) to identify differentially expressed genes in THCA patients (the criteria were an absolute $\log 2$ fold change (FC) $>1$ and adjusted $P$ value $<0.05$ ). Random forest ( $R$ package "ranger") was performed to evaluate the relative importance of various genes in thyroid cancer patients.

\section{Functional enrichment analysis and GSEA analysis}

Kyoto Encyclopedia of Genes and Genomes (KEGG) analysis and Gene Ontology (GO) enrichment analysis were used to confirm the regulatory signaling pathways associated with differentially expressed genes (DEGs) in IHH4 cells with or without WT1 inhibition. We used the "clusterProfiler" R package (version 3.2.11) to identify GO and KEGG signaling pathways. Gene signatures and pathways were obtained from the Molecular Signatures database (MSigDB), GSEA was performed with GSEA software (Version 4.1.0), and $\mathrm{P}<0.05$ was considered statistically significant. 


\section{Cell culture}

BRAF $^{\mathrm{V} 600 E}$ PTC cell lines (including IHH4 cells and BCPAP cells) were purchased from the American Type Culture Collection (ATCC) (Manassas, VA, USA). The IHH4 cells were cultured with a mixture of DMEM and RPMI 1640 in a one-to-one proportion. Then, the mixed medium was supplemented with $2 \mathrm{mM}$ L-glutamine, 10\% fetal bovine serum (FBS) and $100 \mathrm{U} / \mathrm{ml}$ penicillin. The BCPAP cells were cultured in FK12 medium containing $10 \%$ fetal bovine serum (FBS) and $100 \mathrm{U} / \mathrm{mL}$ penicillin. The IHH4 cells and BCPAP cells were incubated at $37^{\circ} \mathrm{C}$ in $5 \% \mathrm{CO}_{2}$ at a $95 \%$ atmosphere at constant temperature.

\section{Cell proliferation assay}

IHH4 cells and BCPAP cells were cultured in 96-well plates (3000 cells per plate in $200 \mu \mathrm{l}$ of medium). Then, $20 \mu \mathrm{l}$ of Cell Counting Kit-8 (CCK-8) reagent (Beyotime, Shanghai, China) was added to every plate and incubated with medium for approximately two hours. The OD450 value was measured with a microplate spectrophotometer (Thermo Fisher Scientific, MA, USA). Cell proliferation was also analyzed with 5-ethynyl-20-deoxyuridine (EdU) agent (Beyotime, Shanghai, China), and the EdU assay was performed according to the manufacturer's protocol. In addition, each experiment was repeated three times.

\section{Transwell assay}

IHH4 cells and BCPAP cells $\left(2 \times 10^{5}\right.$ cells per plate $)$ were cultured in the upper chamber of 24-well culture plates with $8-\mathrm{mm}$-pore membrane inserts. In the upper chamber, $200 \mu \mathrm{l}$ of serum-free medium was added, and $700 \mu \mathrm{l}$ of medium supplemented with FBS was added to the lower chamber. After $24 \mathrm{~h}$, the cells above the membrane in the upper chamber cleaned off the membrane, and cells below the upper chamber membrane were stained with $0.4 \%$ trypan blue. The migrating cells were counted with a light microscope, and each experiment was repeated three times.

\section{Western blot analysis}

Lysates of the IHH4 cells and BCPAP cells were added to SDS-PAGE sample loading buffer (Beyotime, Shanghai, China) and boiled at $100{ }^{\circ} \mathrm{C}$ for 8-10 min. Western blotting was conducted as previously described[24, 25]. The following antibodies were showed in supported information, and anti-actin as an internal control. Protein bands were quantified using Image J software and expressed as fold change with respect to mean control values in the same run (defined as 100 or 1, respectively).

\section{Lentivirus production and transduction}

The WT1-shRNA sequences were cloned into the pLL3.7 vector. The sequences of WT1-shRNA used are shWT1\#1: 5'-CCGGGTGTCTGCTAATGTAAA CTTTCTCGAGAAAGTTTACATTAGCAGACACTT TTTG-3'; shWT1\#2: 5'-CCGGTATAAGTACTAGAT GCATCACCTCGAGGTGATGCATCTAGTACTTAT ATTTTTG-3'. Recombinant lentivirus was generated from 293T cells using calcium phosphate precipitation. IHH4 cells were transfected with lentivirus using polybrene $(8 \mu \mathrm{g} / \mathrm{ml})$ and stable knockdown cells were obtained following selection with $1 \mu \mathrm{g} / \mathrm{ml}$ puromycin for 1 week.

\section{Colony formation survival}

A total of 400 cells were seeded into 6-well in triplicates for plate colony formation survival assay. When visible colonies were formed, cells were fixed after 2 weeks by methanol, stained with $0.2 \%$ crystal violet solution then photographing for colony formation assays.

\section{Cell cycle analysis by flow cytometry}

Seventy percent pre-cooled ethanol was used to fix a total of $1 \times 10^{6}$ cells then washed with PBS and subjected to cell apoptosis analysis using Cell Cycle and Apoptosis Analysis Kit (Yeasen) following the manufacturer's instructions. Data were analyzed by FlowJo v10 software.

\section{Transmission electron microscopy (TEM)}

Cells were fixed with $2 \%$ paraformaldehyde and 2.5\% glutaraldehyde in $0.1 \mathrm{M}$ cacodylate buffer. Cells were then placed in an ice-cold solution of $1 \%$ osmium tetraoxide (Electron Microscopy Sciences) with $0.8 \%$ potassium tetraoxide and $3 \mathrm{mM}$ calcium chloride. Ultrathin sections were prepared and supported on 75 mesh copper grids followed by Sato lead staining.

\section{Nude mouse xenograft models}

Male BALB/c nude mice (between 4 and 6 weeks old) were purchased from HFKbio (Beijing, China) to establish subcutaneous PTC mouse models. IHH4 cells were treated with or without WT1 shRNA and these IHH4 cells $\left(5 \times 10^{6}\right.$ per mouse) were injected subcutaneously into nude mice. After the subcutaneous tumor length and width reached approximately $2.5 \mathrm{~cm} \times 2.5 \mathrm{~cm}$, the tumor volume was measured every two days until the tumor volume was approximately $1000 \mathrm{~cm}^{3}$. All of 
the animal studies were approved by the Institutional Animal Care and Use Committees of Fujian Medical University.

\section{Immunohistochemistry assay}

The tumor tissue from BRAF ${ }^{\mathrm{V} 600 \mathrm{E}}$ PTC xenograft model with or without WT1 knockdown was incubated with $4 \%$ paraformaldehyde for approximately $24 \mathrm{~h}$ and embedded in paraffin. IHC assays were used to measure the protein levels of WT1 and Ki67, and the manufacturer's protocol was constructed as described in a previous study [26]. A primary antibody was used. The integrated optical density (IOD) was quantified using ImageJ software (Version 1.8.0) and the mean optical density (MOD) of each sample was calculated.

\section{Immunofluorescence assay}

IHH4 cells and BCPAP cells were cultured in 6-well plates with cover slips. Then, the cells were placed in $4 \%$ paraformaldehyde for approximately $30 \mathrm{~min}$, and then, $0.03 \%$ Triton X-100 was added and incubated for $20 \mathrm{~min}$. The manufacturer's protocol for the immunofluorescence assay was followed as previously described [44]. The images were analyzed by a fluorescence microscope and ImageJ software (Version 1.8.0).

\section{Statistical analysis}

Statistical analysis of the experimental results was performed using GraphPad Prism software (Version 6.0) and $\mathrm{R}$ software (Version 4.0.3). Differences between two groups were analyzed using Student's t-test, and all results are shown as the means \pm SEM. The statistical significance was set at $\mathrm{P}<0.05$, and each experiment was repeated three times.

\section{Results}

The landscape of mutations and BRAF mutations in thyroid cancer patients

In a previous study, gene mutations were found to play important roles in thyroid cancer progression and development [27]. Additional file 1: Fig. S1A, B indicates the landscape of mutations in thyroid cancer patients as identified through the TCGA database. The analysis showed that BRAF is the most frequently mutated gene and is carried by approximately $60 \%$ of thyroid cancer patients. In thyroid cancer patients, most variant classifications and SNV classes are missense mutations and $\mathrm{C}>\mathrm{T}$ mutations. Figure 1A, B shows the landscape of BRAF mutations in thyroid cancer patients according to the cBioPortal database. Figure $1 \mathrm{C}$ shows that $\mathrm{BRAF}^{\mathrm{VE4} 400}$ is the most common BRAF mutant in thyroid cancer patients. A volcano plot demonstrates the differentially expressed genes (DEGs) in thyroid cancer patients with mutant and wild-type BRAF in the TCGA cohort (Fig. 1D). A gene set enrichment analysis (GSEA) showed the signaling pathways associated with BRAF mutations in thyroid cancer patients. The results verified that "epithelial cell proliferation", "apoptosis" and "selective autophagy" were closely associated with BRAF mutations in thyroid cancer patients (Fig. 1E-G). These

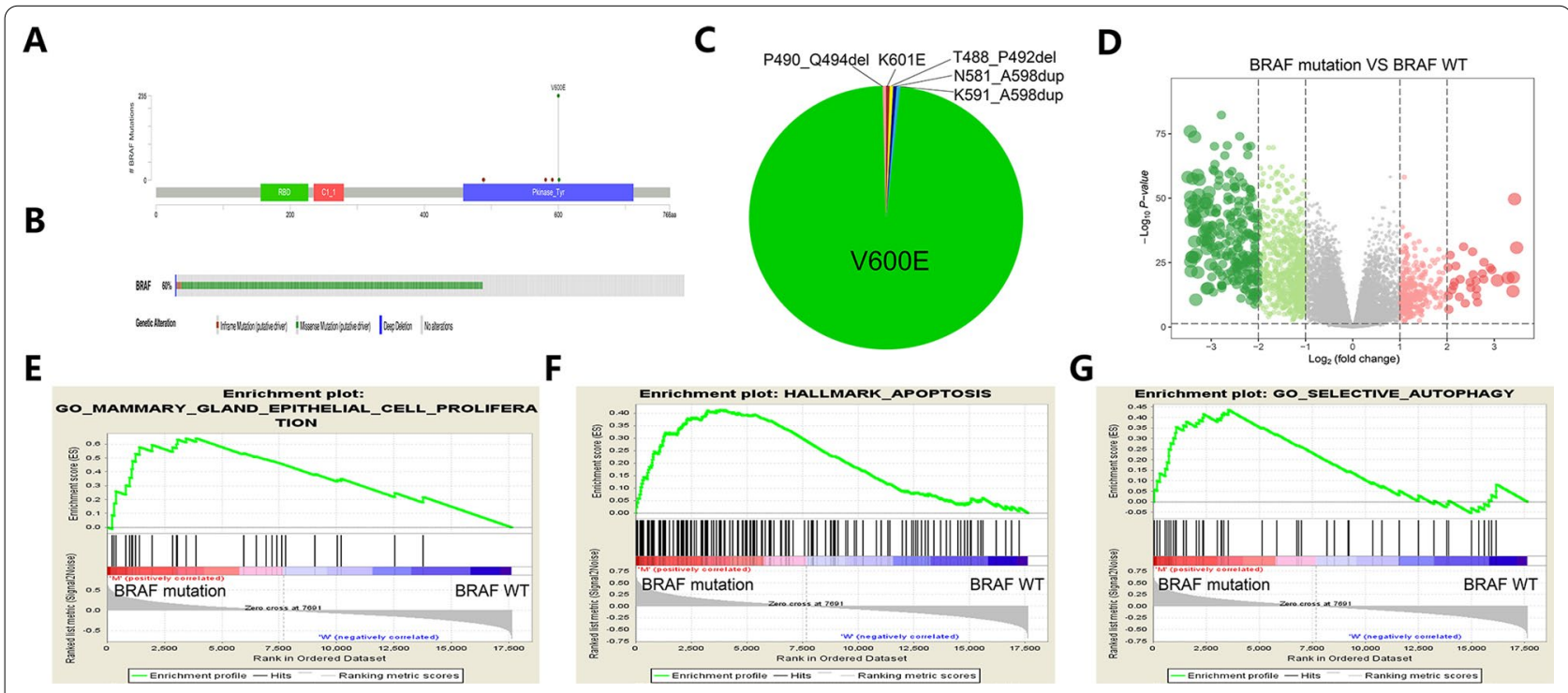

Fig. 1 The landscape of mutations and BRAF mutations in thyroid cancer patients. A, B Somatic mutation of BRAF in thyroid cancer patients. C Changes in BRAF protein expression in thyroid cancer patients. D Differentially expressed genes in thyroid cancer patients carrying mutant BRAF and wild-type BRAF. E-G GSEA showing the upregulated signaling pathways associated with BRAF mutation in thyroid cancer patients 
results suggest that the landscape of mutation and BRAF mutation in thyroid cancer patients and signaling pathways, such as epithelial cell proliferation, apoptosis and autophagy pathways, play critical roles in the progression of thyroid cancer with BRAF mutation.

\section{Identifying the expression and prognostic role of BRAF-activated WT1 in thyroid cancer patients}

A volcano plot indicates the differentially expressed genes (DEGs) between tumor samples and non-tumor samples of thyroid cancer patients (Fig. 2A). We identified prognostic genes by univariate Cox analysis $(P<0.05)$. To further explore the components downstream of activated BRAF, the molecules involved with mutant BRAF and common to the prognostic genes and DEGs were identified in thyroid cancer patients (Fig. 2B). Sixty-seven common genes were selected to perform this analysis. The importance of these 67 genes in thyroid cancer patients was analyzed by random forests. The results showed that WT1 is a significantly important regulator in thyroid cancer patients (Fig. 2C). Figure 2D shows that WT1 was remarkably overexpressed in THCA samples compared with non-tumor samples. Interestingly, WT1 was also upregulated in thyroid cancer samples with mutant BRAF compared with thyroid cancer samples with wildtype BRAF (Fig. 2E). Next, immunohistochemistry (IHC) was used to confirm the expression level of WT1 in PTC tissue. The results implied that WT1 was significantly
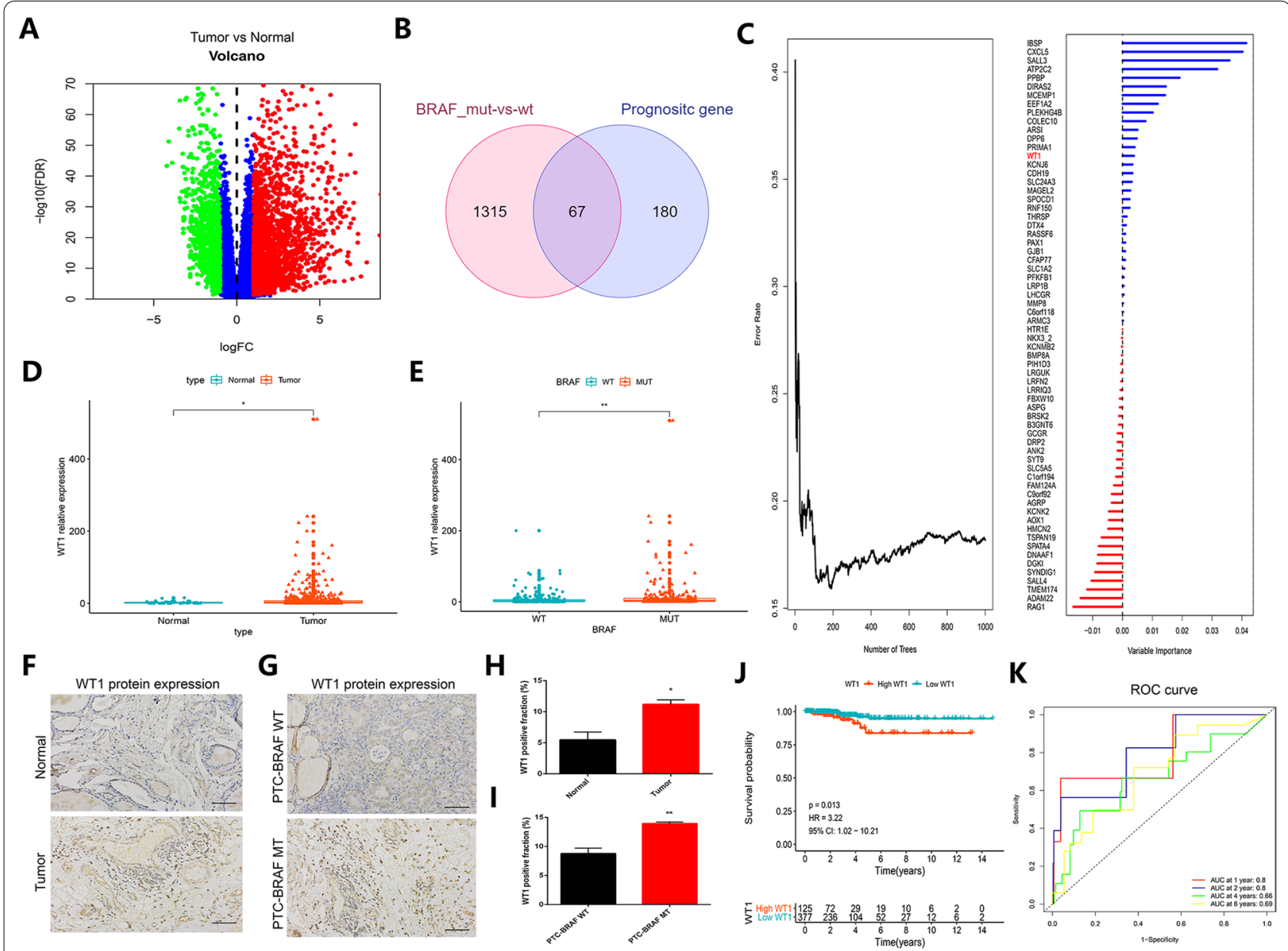

Fig. 2 BRAF-activated WT1 is highly expressed in thyroid cancer patients and predicts poor prognosis. A The differentially expressed genes between thyroid cancer tissues and normal tissues in the TCGA database. B The common regulators among the prognostic genes and DEGs in thyroid cancer patients carrying BRAF-MT and BRAF WT. C The importance of 67 common genes in thyroid cancer patients. D The expression level of WT1 in non-tumor samples compared with that in THCA samples. $\mathbf{E}$ The mRNA level of WT1 in THCA samples with or without BRAF mutation. $\mathbf{F}$ The protein level of WT1 in non-tumor tissues and THCA tumor tissues. G The protein expression of WT1 in THCA patients carrying BRAF WT or BRAF MT. H, I Quantification of immunohistochemistry data of WT1 expression in different groups. J, K KM plot and ROC curve showing the prognostic value of WT1 in thyroid cancer patients $\left({ }^{*} \mathrm{P}<0.05,{ }^{* *} \mathrm{P}<0.01\right)$ 


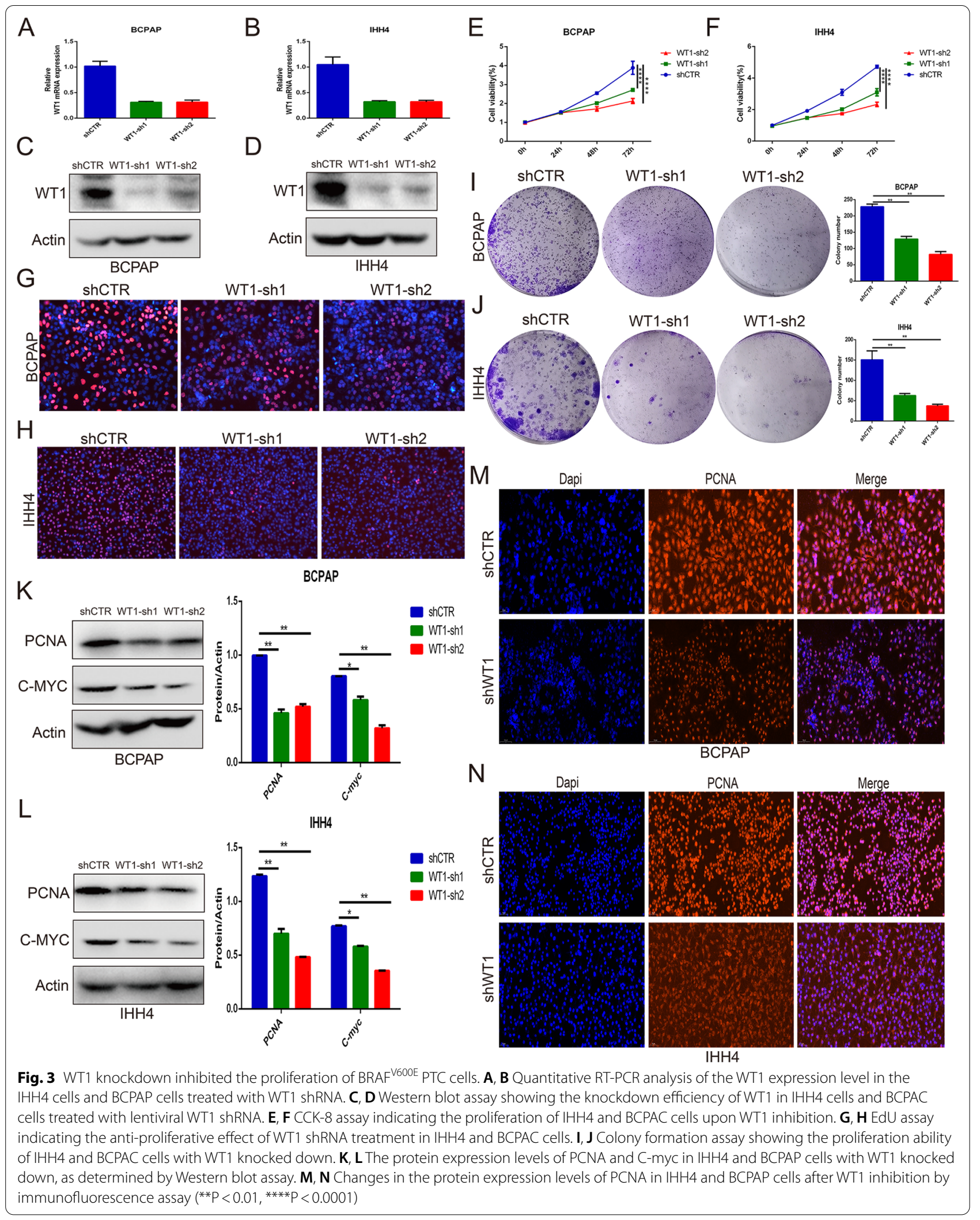




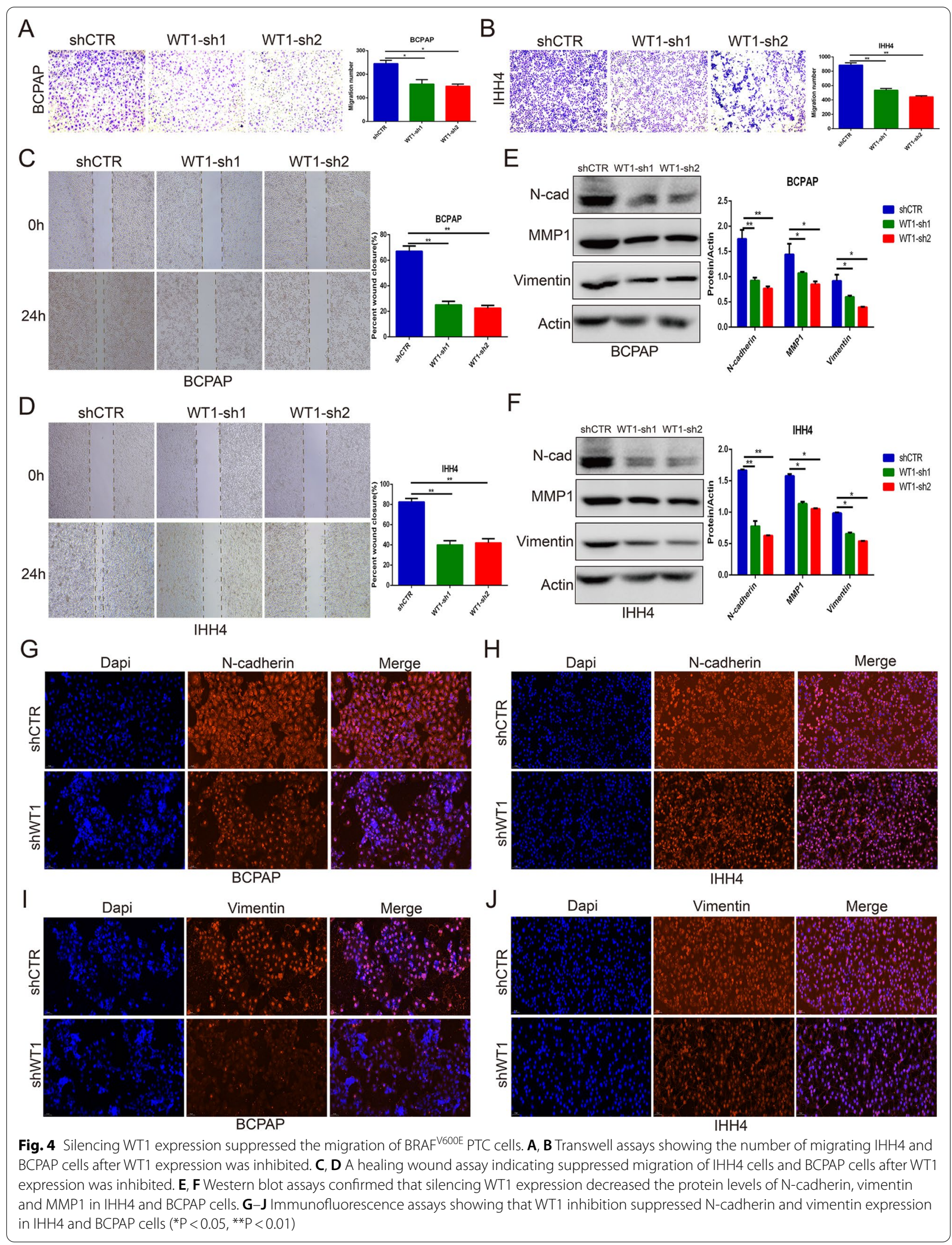


increased in PTC tissue, especially BRAF-mutant PTC tissue (Fig. 2F-I). In addition, higher WT1 expression predicted worse survival time in thyroid cancer patients (Fig. 2J), and the AUC of the ROC curve reached 0.8, $0.8,0.66$ and 0.69 at 1 year, 2 years, 4 years and 8 years, respectively, indicating a superior predictive performance of WT1 for thyroid cancer patients (Fig. 2K). These results suggested that BRAF-activated WT1 may serve as a promising prognostic biomarker in thyroid cancer patients.

\section{Silencing WT1 expression inhibited the proliferation of BRAF ${ }^{\mathrm{V} 600 \mathrm{E}}$ PTC cells}

To explore the role of WT1 in the proliferation and progression of $\mathrm{BRAF}^{\mathrm{V} 600 \mathrm{E}}$ PTC cells, BRAF ${ }^{\mathrm{V} 600 \mathrm{E}} \mathrm{IHH} 4$ cells and BCPAP cells were treated with lentiviral WT1 shRNA. The quantitative RT-PCR and western blot analysis indicated that the expression level of WT1 was remarkably decreased in the IHH4 cells and BCPAP cells treated with WT1 shRNA (Fig. 3A-D). Then, CCK8 , EdU and colony formation assays were performed to measure the proliferation ability of the IHH4 cells and BCPAP cells. The CCK-8 assay indicated that knockdown of WT1 expression significantly suppressed IHH4 and BCPAC cells proliferation (Fig. 3E, F). Similarly, silencing WT1 expression significantly inhibited the proliferation of IHH4 cells and BCPAC cells, as determined by EdU assay (Fig. 3G, H) and colony formation assay (Fig. 3I, J). A western blot analysis showed that WT inhibition notably suppressed the expression levels of PCNA and C-myc in the IHH4 and BCPAP cells (Fig. 3K, L). In addition, immunofluorescence assays implied that the knockdown of WT1 expression effectively decreased the expression of PCNA in the IHH4 cells and BCPAP cells (Fig. 3M, N). These results suggest that WT1 may play an oncogenic role in the proliferation of $\mathrm{BRAF}^{\mathrm{V} 600 \mathrm{E}} \mathrm{PTC}$ cells, namely, IHH4 and BCPAP cells.

\section{Gene knockdown of WT1 inhibited the migration of BRAF ${ }^{\mathrm{V} 600 \mathrm{E}}$ PTC cells}

To investigate the function of WT1 in regulating $\mathrm{BRAF}^{\mathrm{V} 600 \mathrm{E}}$ PTC cell migration, Transwell assays and wound healing assays were performed with $\mathrm{IHH} 4$ and BCPAP cells. WT1 inhibition remarkably suppressed the migration of IHH4 and BCPAP cells, as indicated by Transwell assay (Fig. 4A, B) and healing wound assay (Fig. 4C, D). Western blot and immunofluorescence assays were also performed to measure the protein levels of proteins involved in the migration process, such as N-cadherin, Vimentin and MMP1. The western blot analysis indicated that knockdown of WT1 expression effectively decreased the protein levels of N-cadherin, Vimentin and MMP1 in IHH4 cells and BCPAP cells (Fig. 4E, F). Similarly, immunofluorescence assays showed that silencing WT1 expression downregulated the protein levels of N-cadherin and Vimentin in IHH4 cells and BCPAP cells (Fig. 4G-J). All the results indicated that WT1 gene knockdown inhibited the migration of BRAF ${ }^{\mathrm{V} 600 \mathrm{E}}$ PTC cells.

\section{WT inhibition suppressed autophagy and promoted apoptosis of BRAF ${ }^{\mathrm{V} 600 \mathrm{E}}$ PTC cells}

To explore the biological process of WT1 in BRAF ${ }^{\mathrm{V} 600 \mathrm{E}}$ PTC progression and development, Transmission electron microscopy (TEM) assays indicated that the number of autophagic vacuoles (autophagosomes and autolysosomes) was significantly reduced in $\mathrm{IHH} 4$ and BCPAP cells after WT1 inhibition (Fig. 5A, B). Flow cytometry analysis showed that silencing WT1 expression significantly induced apoptosis of the IHH4 cells and BCPAP cells (Fig. 5C, D).Interestingly, knockdown of WT1 expression notably increased the protein level of P62 and LC3A/B and downregulated the protein levels of ATG5 and ATG7 in the IHH4 cells and BCPAP cells (Fig. 5E, F, I J). A western blot analysis demonstrated that WT1 inhibition effectively enhanced the protein expression of cleaved caspase 3 , and BAX and suppressed the protein expression level of BCL2 in the IHH4 cells and BCPAP cells (Fig. 5G-J). These results demonstrate that the altered expression of WT1 leads to changes in autophagy and apoptosis pathways in BRAF ${ }^{\mathrm{V} 600 \mathrm{E}}$ PTC cells.

\section{WT1 activated the AKT/mTOR signaling pathway and ERK/ P65 signaling pathway in BRAF ${ }^{\mathrm{V} 600 \mathrm{E}}$ PTC cells}

The molecular mechanism of WT1 in the progression of $\mathrm{BRAF}^{\mathrm{V} 600 \mathrm{E}}$ PTC cells was investigated, and the results are presented as a volcano plot showing the differentially

\footnotetext{
(See figure on next page.)

Fig. 5 Gene knockdown of WT1 regulated autophagy and apoptosis of BRAF ${ }^{\mathrm{V} 600 \mathrm{E}}$ PTC cells. A, B Transmission electron microscopy was performed to observe the numbers of autophagic vacuoles, including autophagosomes and autolysosomes, in IHH4 and BCPAP cells after WT1 inhibition. C, D Flow cytometry analysis showing that WT1 inhibition promoted the apoptosis of IHH4 and BCPAP cells. E, F Western blot analysis showing the protein changes in LC3A/B, P62, ATG5 and ATG7 in IHH4 and BCPAP cells with WT1 knocked down. G, H Western blot assay showing that WT1 knockdown modulated the protein expression levels of cleaved caspase $3, \mathrm{BCL} 2$ and BAX in IHH4 and BCPAP cells. I, J Quantification of protein expression of ATG7, ATG5, P62, LC3A/B, BCL2, BAX and cleaved caspase 3 in WT1-silencing IHH4 cells and BCPAP cells (*P<0.05, ${ }^{* * * P}<0.001$ )
} 

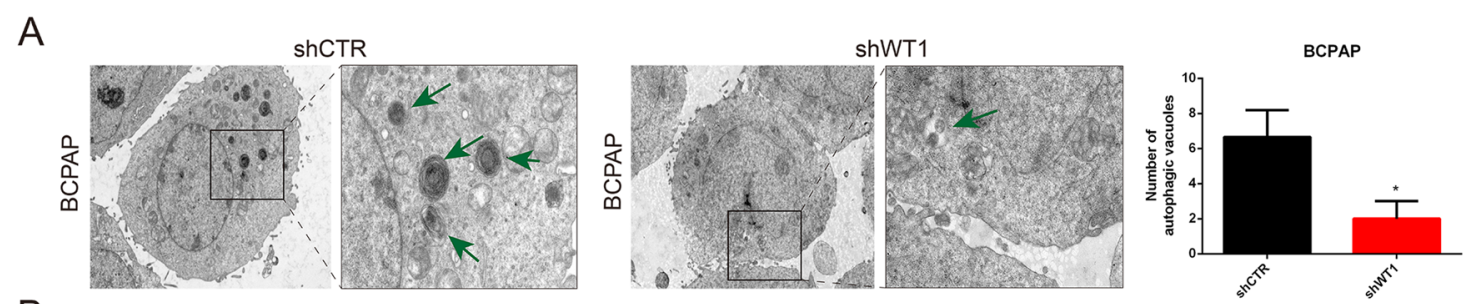

B

ShCTR
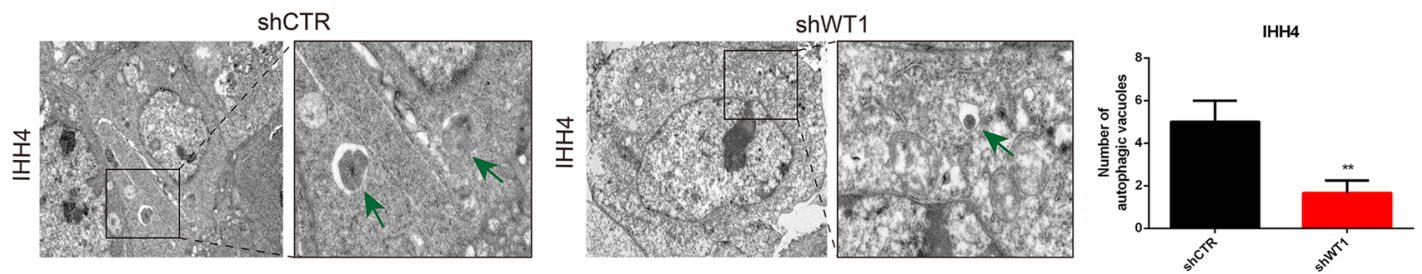

C
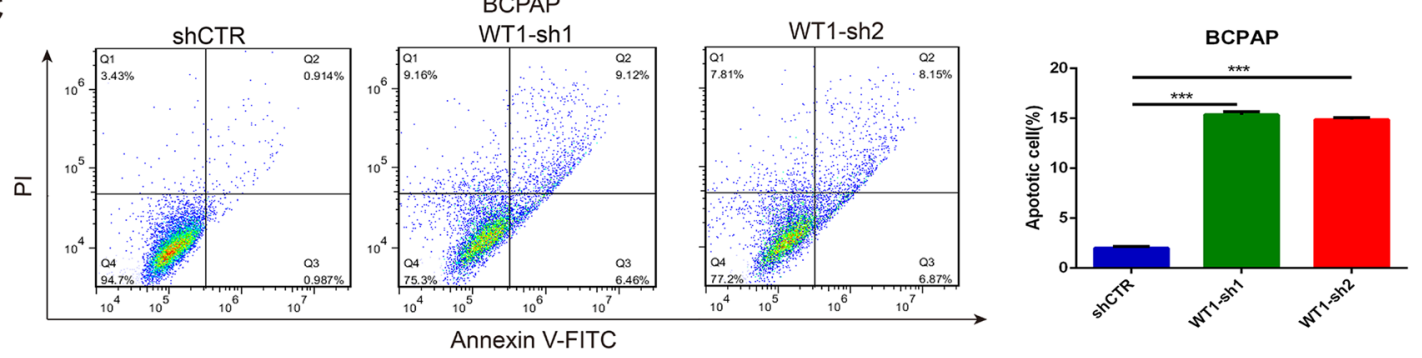

D
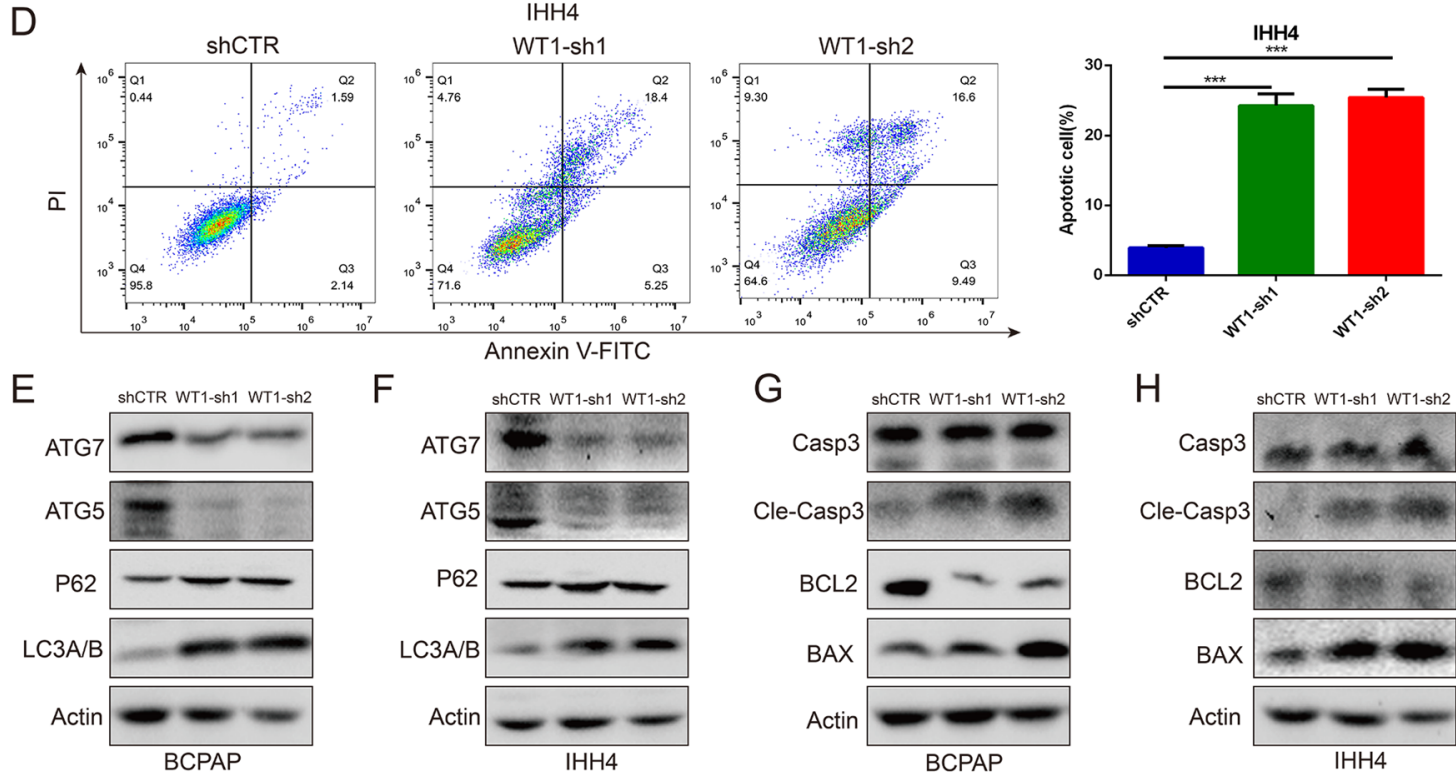

F
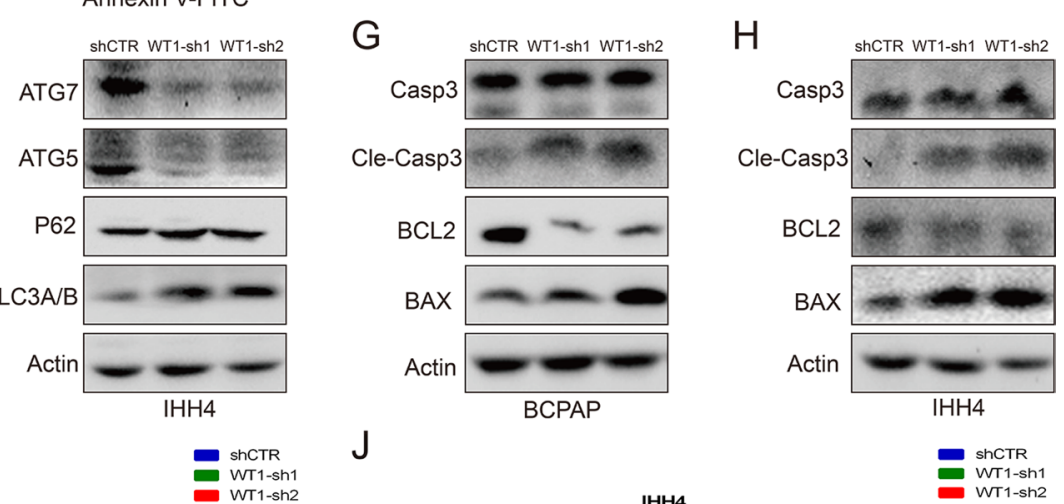

I shCTR
W1-1-h1
WT1-sh2
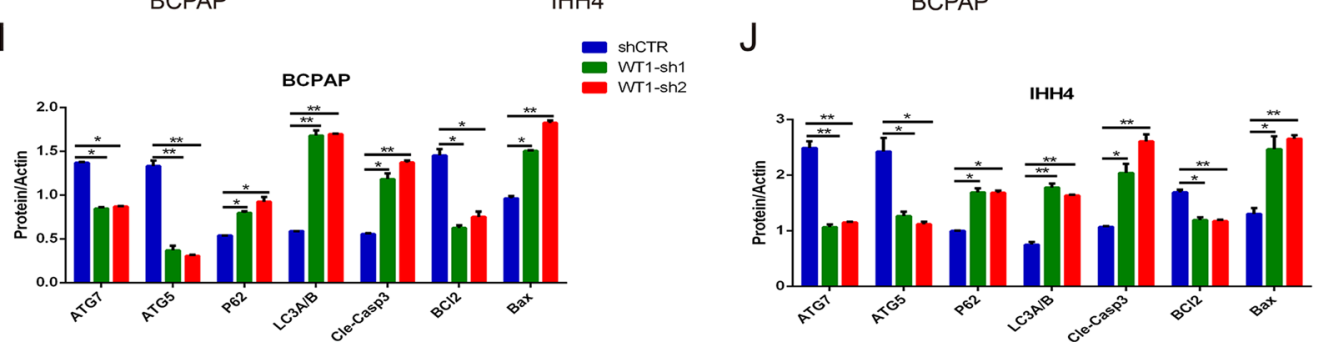

Fig. 5 (See legend on previous page.) 
expressed genes (DEGs) in IHH4 cells with or without WT1 knocked down (Fig. 6A). A Kyoto Encyclopedia of Genes and Genomes (KEGG) analysis indicated that WT1 is associated with the "PI3K/AKT signaling network", "mTOR signaling cascades", "MAPK signaling pathway" and "NF-kappa B signaling pathway" in PTC cells (Fig. 6B). Top 20 go functional analysis indicated "epithelial cell differentiation" and "transmembrane receptor protein serine/threonine kinase signaling pathway" is changed in IHH4 cells with WT1 knocked down (Fig. 6C). Then, a protein-protein interaction (PPI) network was generated using the GeneMANIA database, and it showed the potential regulatory proteins of WT1 (Fig. 6D). Interestingly, A GSEA indicated that WT1 is involved in the "MAPK signaling pathway" and "mTOR signaling pathway" in thyroid cancer patients (Fig. 6E, F). A western blot analysis revealed that knockdown of WT1 expression effectively inhibited the protein expression levels of phosphorylated ERK and phosphorylated P65 in IHH4 and BCPAP cells (Fig. 6G, H). In addition, WT1 inhibition also suppressed the protein levels of phosphorylated AKT, phosphorylated mTOR, and phosphorylated $\mathrm{S} 6$ in IHH4 and BCPAP cells (Fig. 6I, J). However, the total protein level of these molecules was not changed in the IHH4 cells or BCPAP cells (Fig. 6G-L). All these results suggest that $\mathrm{WT} 1$ activated $\mathrm{AKT} / \mathrm{mTOR}$ cascades and the ERK/P65 signaling axis in BRAF ${ }^{\mathrm{V} 600 \mathrm{E}} \mathrm{PTC}$ cells.

\section{Knockdown of WT1 expression inhibited the growth of PTC tumors expressing BRAF ${ }^{\mathrm{V} 600 \mathrm{E}}$}

To confirm the roles of WT1 in tumor growth of BRAF $^{\mathrm{V} 600 \mathrm{E}}$ PTC cells, IHH4 cells were treated with lentiviral WT1 shRNA to stably knockdown WT1 expression, and western blotting was performed to measure the WT1 knockdown efficiency in the IHH4 cells (Fig. 7A). Then, IHH4 cells with or without WT1 were subcutaneously injected into male BALB/c nude mice to establish subcutaneous BRAF ${ }^{\mathrm{V} 600 \mathrm{E}}$ PTC xenograft models. Images revealed that knockdown of WT1 expression effectively abolished the growth of BRAF ${ }^{\mathrm{V} 600 \mathrm{E}}$ PTC tumors (Fig. 7B). Figure 7C-E shows that silencing WT1 expression notably reduced the tumor volume (Fig. 7C), tumor weight (Fig. 7D) and tumor load (Fig. 7E) in BRAF ${ }^{\mathrm{V} 600 \mathrm{E}}$ PTC.
HE staining implied that the pathological characteristics of subcutaneous BRAF ${ }^{\mathrm{V} 600 \mathrm{E}}$ PTC xenograft tissue with or without WT1 knockdown (Fig. 7F). In addition, IHC assays showed that the protein levels of Ki67, N-cadherin, ATG7, P-Akt and P-ERK were remarkably decreased and that the protein level of cleaved caspase 3 was upregulated upon WT1 inhibition (Fig. 7G-N). Altogether, these results demonstrate that knocking down the WT1 gene significantly suppressed tumor progression and the development of BRAF ${ }^{\mathrm{V} 600 \mathrm{E}}$ PTC.

\section{Validation of the independent predictive ability of WT1 in thyroid cancer patients}

To determine the predictive value of WT1 in thyroid cancer patients, univariate and multivariate Cox regression analyses were performed to identify the independent prognostic factors through forest plots. The results revealed that TNM stage and WT1 expression levels can be regarded as independent prognostic factors in thyroid cancer patients in the TCGA-THCA cohort (Fig. 8A). Then, TNM stage and WT1 expression levels were used to construct a predictive nomogram of thyroid cancer patients in the TCGA-THCA cohort (Fig. 8B). The calibration curves indicated that the nomogram showed great consistency in predicting overall survival rates at 1,3 and 5 years (Fig. 8C-E). Sankey plot indicated the relationship between TNM stage, BRAF mutated status and WT1 expression in thyroid cancer patients (Fig. 8F). Next, the prognotic role of WT1 was assessing between BRAF mutated and BRAF wild-type thyroid cancer patients. There was no significant change in survival time compared with high WT1 expression and low WT1 expression in BRAF wild-type thyroid cancer patients (Fig. 8G). However, The KM plot showed higher expression level of WT1 predicted worse overall survival time in thyroid cancer patients carrying BRAF mutation (Fig. 8H). And schematic of mechanism of BRAF-activated WT1 contributes to cancer growth and regulates autophagy and apoptosis in papillary thyroid carcinoma (Fig. 8I). These results suggest that WT1 may function as an independent prognostic factor and is closely associated with the survival time of thyroid cancer patients carrying $\mathrm{BRAF}^{\mathrm{V} 600 \mathrm{E}}$.

(See figure on next page.)

Fig. 6 WT1 activated the AKT/mTOR signaling pathway and ERK/P65 signaling pathway in BRAF ${ }^{\mathrm{V} 600 \mathrm{E}}$ PTC cells. A The differentially expressed genes (DEGs) in IHH4 cells treated with vector lentivirus and shWT1 lentivirus. B KEGG analysis indicating the signaling pathways associated with DEGs in $\mathrm{IHH} 4$ cells. C Go functional analysis showed the 20 most regulatory signaling pathways associated with DEGs in IHH4 cells by Metascape database. D The protein-protein interaction (PPI) network indicated the directly regulated molecules of WT1, as determined with the GeneMANIA database. E, F GSEA showing that higher WT1 expression led to upregulated signaling pathways in thyroid cancer patients. G-J Western blot analysis showing that WT1 silencing decreased the essential components of AKT/mTOR cascades and the ERK/P65 signaling pathway in IHH4 and BCPAP cells. $\mathbf{K}, \mathbf{L}$ Quantification of protein expression of the essential components of AKT/mTOR cascades and the ERK/P65 signaling pathway in WT1-silencing IHH4 and BCPAP cells 

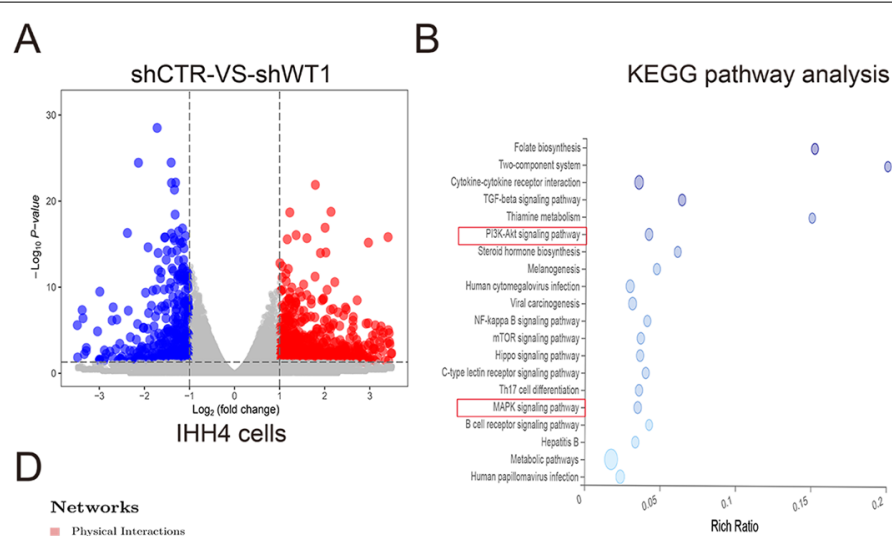

C

GO pathway analysis

Networks

II Physical Interaction

= Co-expression

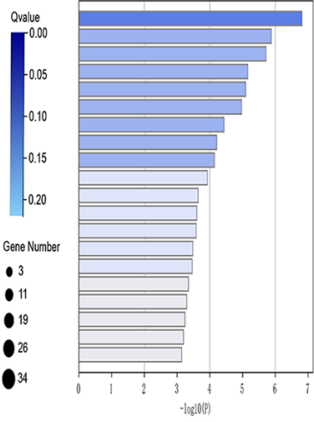

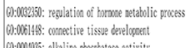

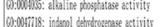

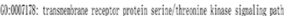

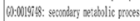

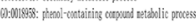

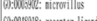

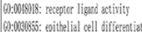

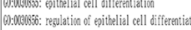

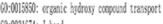

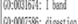

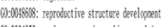

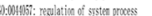

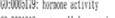

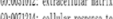

In Predicted

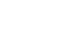

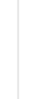

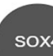

sox4

CIAO1

SLCGAO

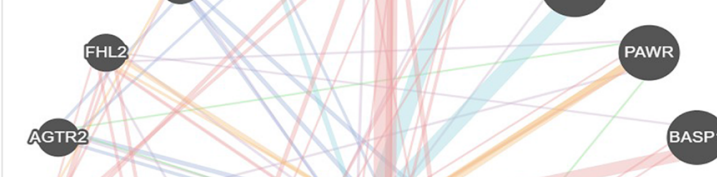

whit

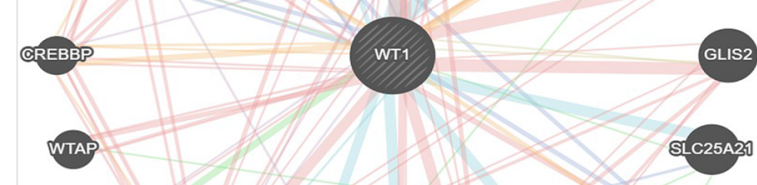

PAX8

PAX2

Mro

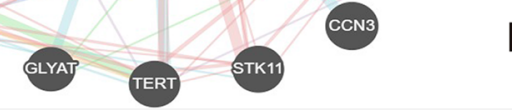

G

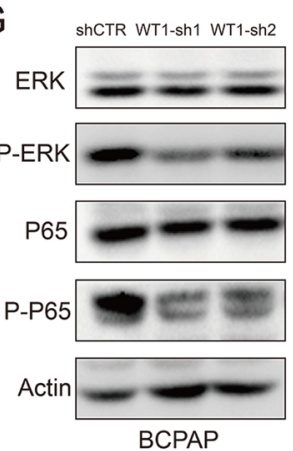

$\mathrm{K}$
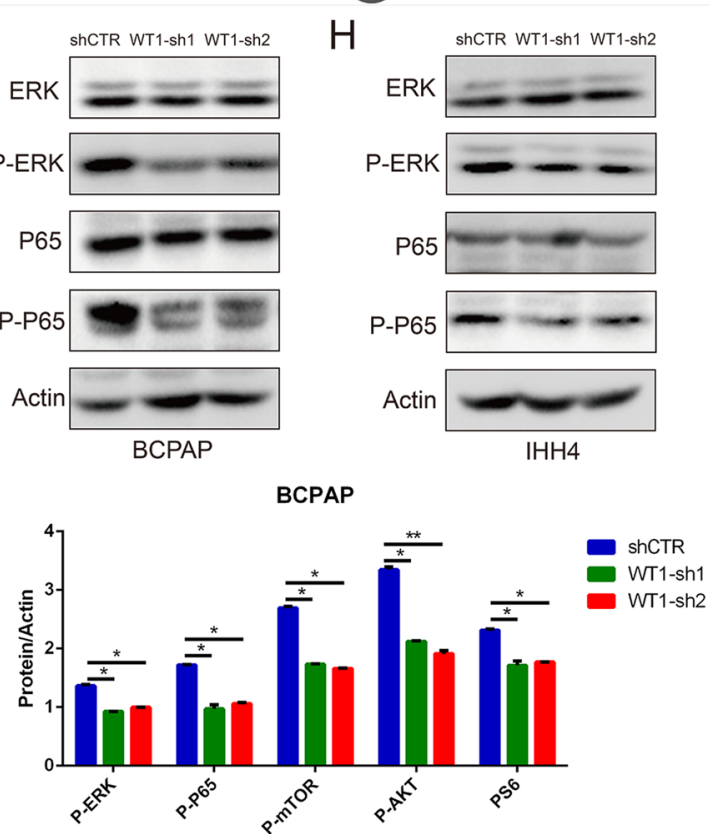

E
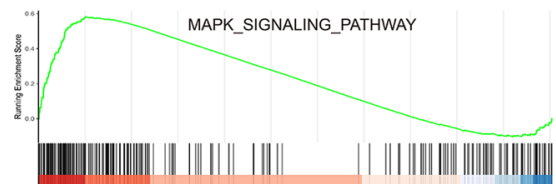

High WT1 expression Low WT1 expression

F

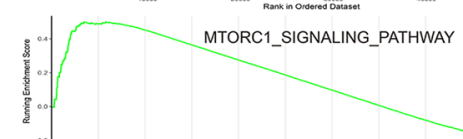

"|||||||||||||||||||||| | || |||| | | | | | | || |||||||||||||||||||||||||
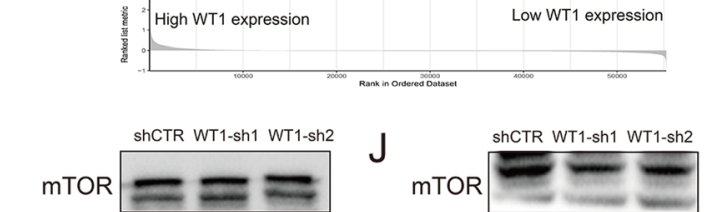

P-mTOR $=$

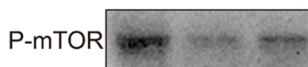
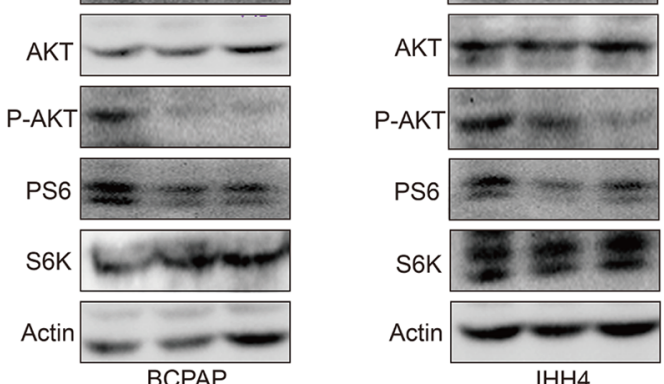

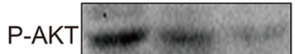

PS6 tere mane ner

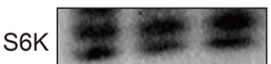

Actin

$\mathrm{IHH} 4$

L

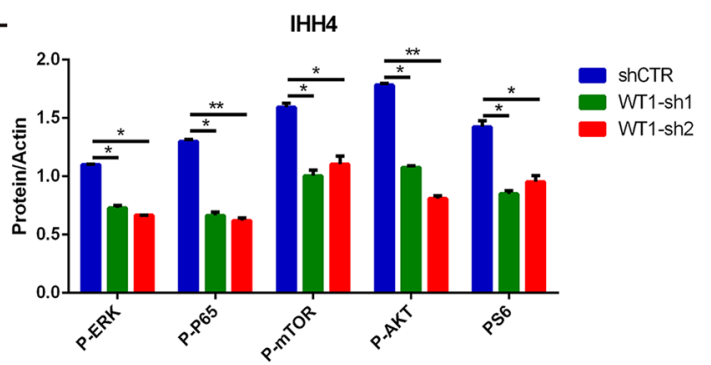

Fig. 6 (See legend on previous page.) 


\section{Discussion}

Thyroid papillary carcinoma is a kind of endocrine malignancy, and the morbidity of PTC is gradually increasing. According to previous study, the PTC incidence was 1.80 per 100,000 males and 6.20 per 100,000 in females worldwide [28]. Despite the improvement in current treatments, patients with PTC tend to develop distant metastases or recurrence and have poor prognosis because of complex biological characteristics and unclear pathological mechanisms [29]. In this study, we found that WT1 is highly expressed in PTC patients, and higher WT1 expression is predictive of worse overall survival time. In addition, WT1 also functioned as an independent prognostic factor with high predictive value. This result may help improve the clinical outcomes for PTC patients and indicates possibilities for personalized treatment. BRAF mutations are the most frequent mutation subtypes in PTC patients, and targeting BRAF mutants to develop targeted therapy drugs may be an effective method to overcome PTC [30]. In this study, we comprehensively analyzed the differentially expressed genes in BRAF MT and BRAF WT PTC tissue samples obtained from a TCGA patient cohort. Then, we identified the signaling pathway in PTC patient cells with BRAF mutations. The results indicated that BRAF mutation is closely related to the "epithelial cell proliferation", "apoptosis" and "selective autophagy" signaling pathways in PTC patients, as determined through a GSEA.

WT1 encodes a zinc-finger transcription factor that also plays oncogenic or tumor suppressor roles at the transcriptional and post-transcriptional levels in various malignancies [15]. The expression level of WT1 is remarkably increased in primary thyroid cancer, and WT1 is regarded as a critical prognostic biomarker related to recurrence-free survival in thyroid cancer patients [31, 32]. However, the regulatory relationship between activated BRAF and WT1 remains unclear. The role of WT1 in BRAF mutated PTC progression and development also needs to be further explored. In this study, we found that WT1 was activated downstream of BRAF in PTC patients. More importantly, knockdown of WT1 expression notably reduced the proliferation and migration of BRAF ${ }^{\mathrm{V} 600 \mathrm{E}}$ PTC cells. Silencing WT1 also remarkably decreased the expression levels of PCNA,
C-myc and N-cadherin in BRAF ${ }^{\mathrm{V} 600 \mathrm{E}}$ PTC cells. In addition, WT1 inhibition significantly suppressed tumor growth of $\mathrm{BRAF}^{\mathrm{V} 600 \mathrm{E}} \mathrm{PTC}$ cells in vivo. These results indicate the important role of WT1 in cancer progression and the development of PTC, in particular, and inhibiting WT1 may serve as a promising treatment strategy against PTC with BRAF ${ }^{\mathrm{V} 600 \mathrm{E}}$.

Autophagy is an evolutionarily conserved pathway that ultimately leads to intracellular protein and organelle degradation in lysosomes and is triggered by stress and nutrient deprivation [33]. Apoptosis, also called programmed cell death, is a highly ordered and orchestrated cell death process [34]. Autophagy and apoptosis are two different kinds of programmed cell death and play critical roles in tumorigenesis and cancer progression [35]. In a previous study, triggering apoptosis inhibited cancer cell survival and proliferation [36]. In this study, we found that BRAF mutation is closely associated with "autophagy" and "apoptosis" signaling pathways. WT1 was confirmed as a downstream molecule of BRAF and silencing WT1 expression remarkably promoted apoptosis and inhibited autophagy in PTC cells. These results indicated the oncogenic role of $\mathrm{WT} 1$ in regulating the cell death process in BRAF ${ }^{\mathrm{V} 600 \mathrm{E}}$ PTC cells. Akt, also known as the PKB (protein kinase B) and mTOR (mammalian target of rapamycin) signaling pathway, is the most frequently activated signaling network in human cancers [37, 38]. Extracellular signal-regulated kinase (ERK) and nuclear factor kappa B (NF-kB) also play essential roles in many biological processes, such as cell growth, tumorigenesis and autophagy and apoptosis [39-42]. More importantly, in a previous study, the BRAF ${ }^{\mathrm{V} 600 \mathrm{E}}$ mutant notably activated the MAPK signaling pathway in BRAF-mutant thyroid cancers [43, 44]. In this study, RNA sequence revealed that WT1 is closely associated with PI3K/AKT signaling network, mTOR signaling cascades, MAPK signaling pathway and NF-kappa B signaling pathway. Experiments implied that silencing WT1 expression effectively decreases AKT phosphorylation, mTOR phosphorylation and the downstream protein levels of the mTOR signaling pathway (including S6 phosphorylation) in BRAF ${ }^{\mathrm{V} 600 \mathrm{E}}$ PTC cells. Interestingly, WT1 inhibition significantly decreased the protein level of phosphorylated ERK and phosphorylated P65

(See figure on next page.)

Fig. 7 WT1 inhibition attenuates tumor growth of BRAF ${ }^{\mathrm{V} 600 \mathrm{E}}$ mutation PTC. A Western blot analysis indicated the stable inhibition efficiency of lentiviral WT1 shRNA treatment. B The image revealed the tumor volume in the control vector group and WT1 shRNA group. C The tumor volume of BRAF ${ }^{\mathrm{V} 600 \mathrm{E}}$ PTC xenografts was measured every three days. D, E The tumor weight and tumor load of BRAF ${ }^{\mathrm{V} 600 \mathrm{E}} \mathrm{PTC}$ xenografts were determined when tumor-bearing mice were sacrificed. $\mathbf{F}$ HE staining indicated the pathological features of BRAF ${ }^{\mathrm{V} 600 \mathrm{E}}$ PTC xenografts tissue with or wihout WT1 inhibition. G-L IHC analysis demonstrating that WT1 knockdown regulated the protein expression of Ki67, N-cadherin, ATG7, cleaved caspase 3, P-Akt and P-ERK in BRAF V600E PTC xenograft tissues. M, N Quantification of immunohistochemistry data of the protein expression of Ki67, N-cadherin, ATG7,cleaved caspase 3, P-Akt and P-ERK in BRAFV600E PTC xenograft tissues. $\left({ }^{* * *} \mathrm{P}<0.001\right.$, $\left.{ }^{* * * *} \mathrm{P}<0.0001\right)$ 

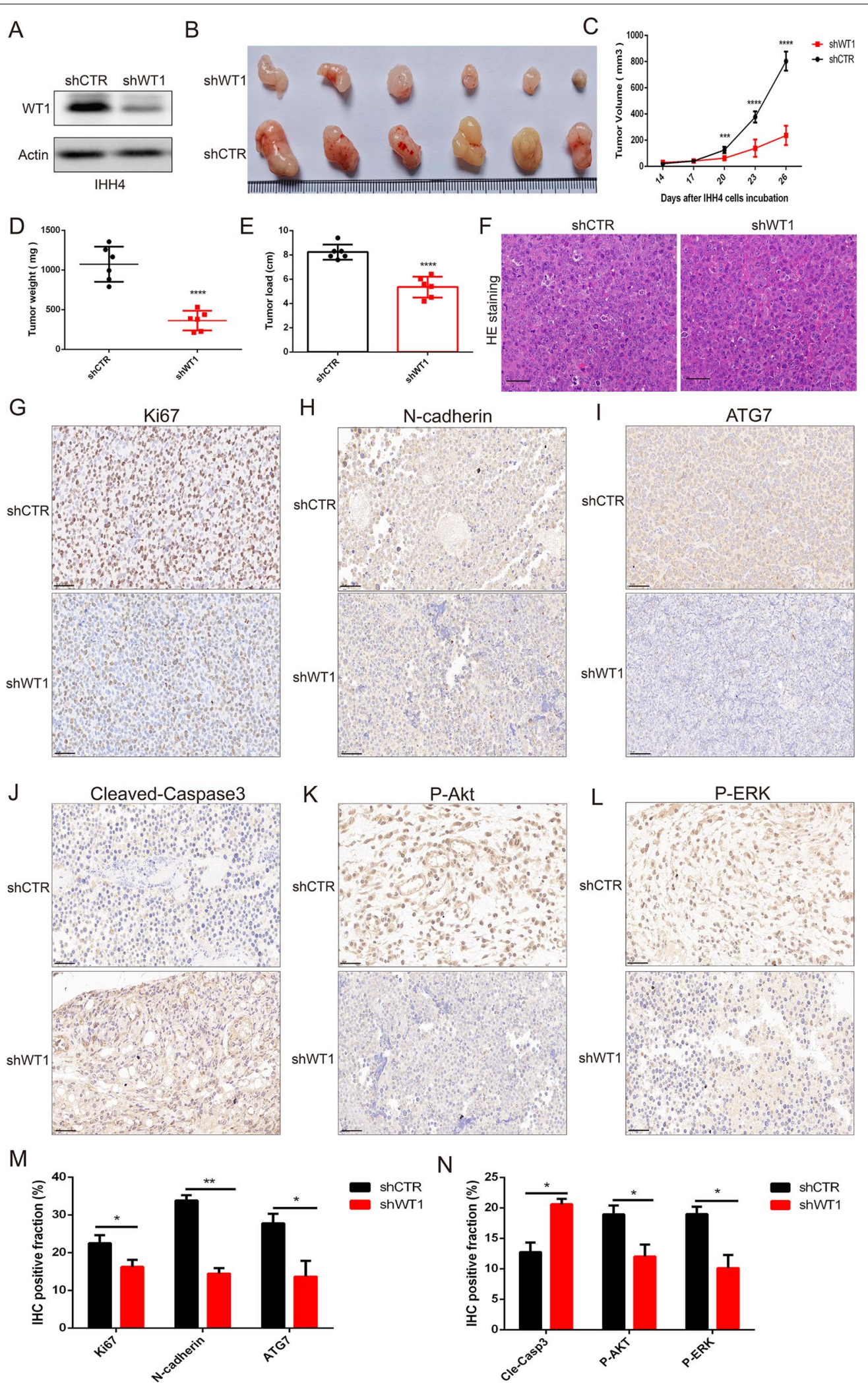

Fig. 7 (See legend on previous page.) 


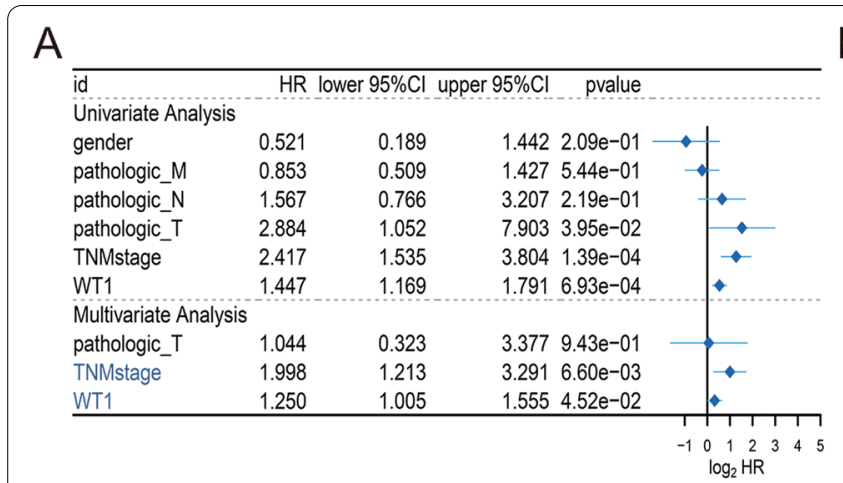

C

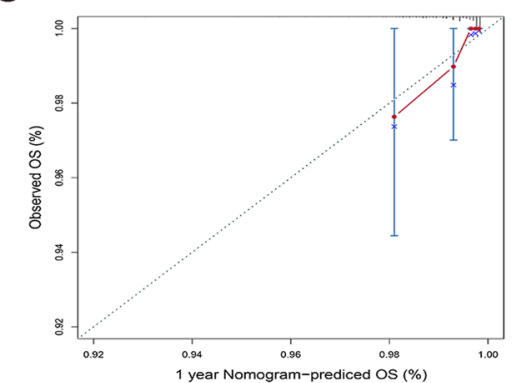

B

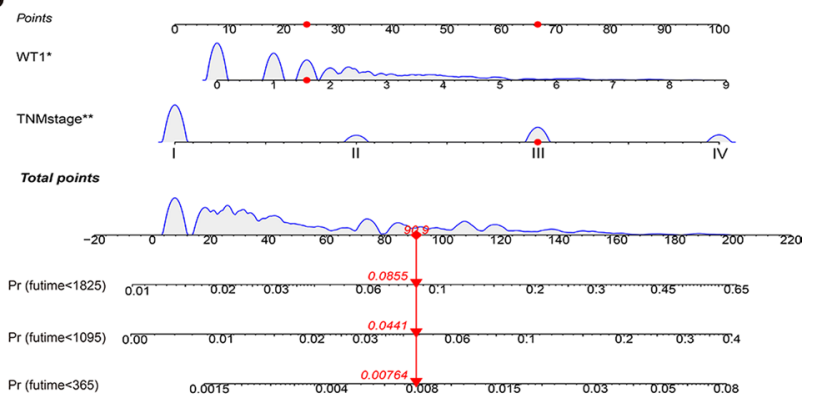

E
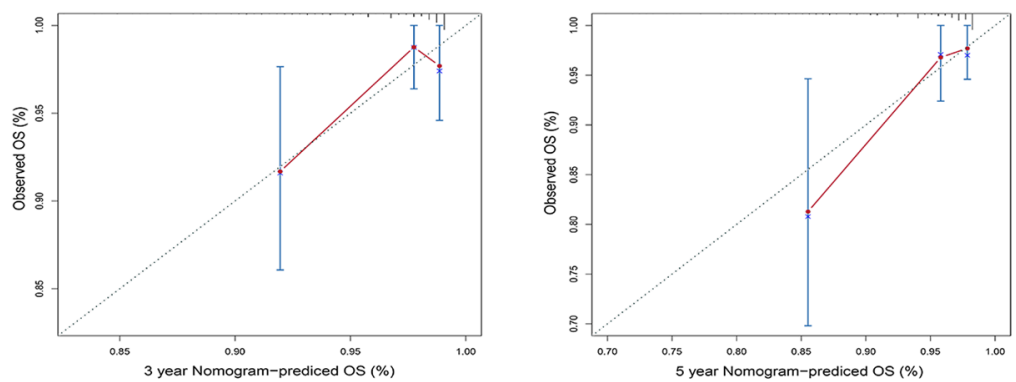

$\mathrm{F}$

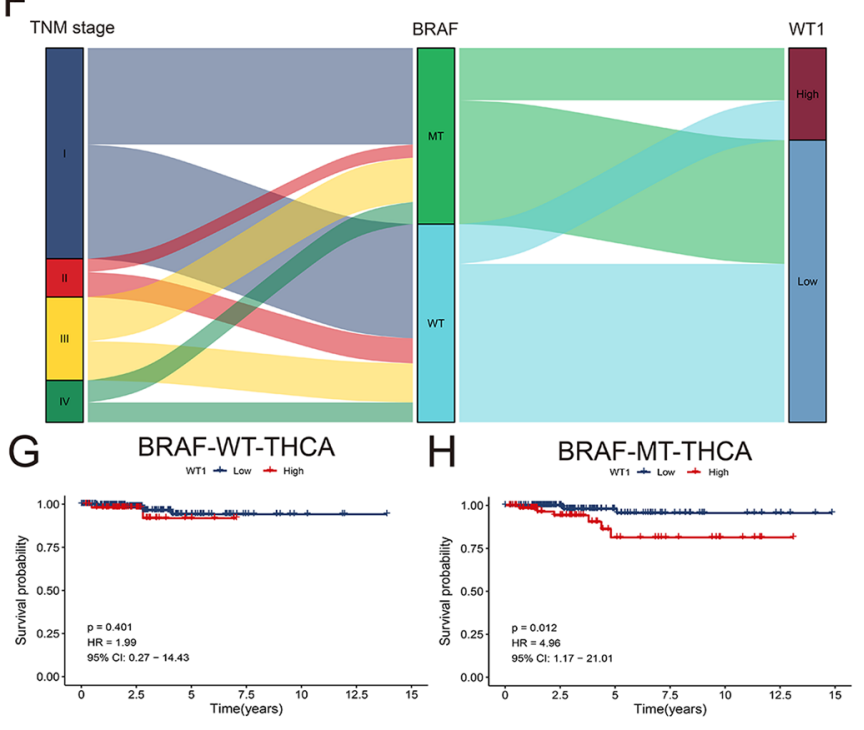

I

BRAF mutation papillary thyroid carcinoma

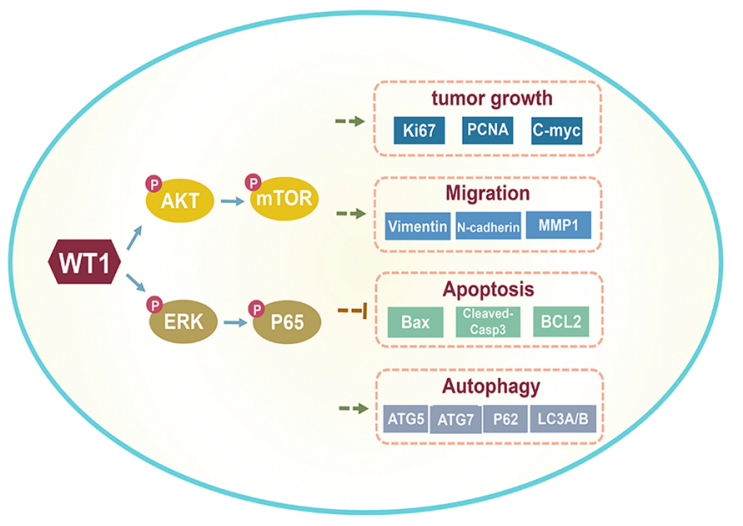

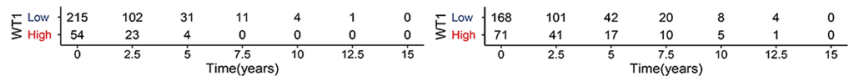

Fig. 8 Validation of the independent predictive ability of WT1 in thyroid cancer patients. A Forest plot showing the prognostic performance of WT1 and other clinical features in THCA cohort patients in the TCGA database. B The nomogram showing the predicted overall survival rate of thyroid cancer patients at 1 year, 3 years and 5 years on the basis of independent prognostic factors including TNM stage and WT1 expression level. C-E Calibration curves revealing superior consistency of the predictive nomogram model at 1 year, 3 years and 5 years. $\mathbf{F}$ The relationship between WT1 expression, BRAF mutated status and TNM stage in thyroid cancer patients. G, H K-M survival analysis implied the prognostic value of WT1 in thyroid cancer patients carrying BRAF mutation or BRAF wild-type. I Schematic of mechanism of BRAF-activated WT1 contributes to cancer growth and regulates autophagy and apoptosis in papillary thyroid carcinoma

in $\mathrm{BRAF}^{\mathrm{V} 600 \mathrm{E}} \mathrm{PTC}$ cells. In summary, WT1 contributes to cell proliferation, migration and growth of papillary thyroid carcinoma through augmented activation of the AKT/mTOR axis and ERK/p65 signaling pathway.
In summary, we identified differentially expressed genes and signaling pathways in thyroid cancer patients carrying mutant BRAF. Then, we confirmed that higher WT1 levels were closely related to worse prognosis in 
thyroid cancer patients with BRAF mutation. In addition, knockdown of WT1 expression remarkably inhibited the proliferation and migration of $\mathrm{BRAF}^{\mathrm{V} 600 \mathrm{E}} \mathrm{PTC}$ cells. WT1 inhibition significantly triggered cell apoptosis and inhibited autophagy in BRAF ${ }^{\mathrm{V} 600 \mathrm{E}}$ PTC cells. More importantly, silencing WT1 expression effectively inhibited AKT/mTOR and ERK/P65 signaling in BRAF ${ }^{\mathrm{V} 600 \mathrm{E}}$ PTC cells. All these results highlight the biological functions of WT1 and suggest a novel therapeutic strategy for PTC with BRAF ${ }^{\mathrm{V} 600 \mathrm{E}}$.

However, there are also some limitations in the current study. First, the RNA sequencing, mutation data and clinical information were obtained primarily from the TCGA database, and more clinical samples are needed to validate the prognostic role of WT1 in BRAF mutated PTC patients. Second, more experiments are needed to confirm the biological functions of WT1 and BRAF ${ }^{\mathrm{V} 600 \mathrm{E}}$ in PTC, such as experiments with mice with WT1 conditionally knocked out. Third, the WT1 expression in the BRAF mutated background reduced survival but not in the non-mutated BRAF background, which has aroused our great interest and therefore required further experimental research. Need to be explored in further studies.

\begin{abstract}
Abbreviations
THCA: Thyroid carcinoma; PTC: Papillary thyroid carcinoma; WT1: Wilms'tumor 1; DEGs: Differentially expressed genes; TCGA: The Cancer Genome Atlas; KEGG: Kyoto Encyclopedia of Genes and Genomes; GO: Gene ontology; FBS: Fetal bovine serum; IHC: Immunohistochemistry; ROC: Receiver operating characteristic curve; PKB: Protein kinase B; mTOR: Mammalian target of rapamycin; PKB: Protein kinase B; ERK: Extracellular signal-regulated kinase; NF-kB: Nuclear factor kappa B.
\end{abstract}

\section{Supplementary Information}

The online version contains supplementary material available at https://doi. org/10.1186/s12967-022-03260-7.

Additional file 1: Figure S1. The landscape of mutations in thyroid cancer patients as identified through the TCGA database. (A) The 15 most frequently mutated genes in thyroid cancer patients in the TCGA database.

(B) The variant classification, variant type, snv class, variant per case and 10 most frequently mutated genes in thyroid cancer patients.

\section{Acknowledgements}

The authors would like to acknowledge the helpful comments regarding this paper received from the reviewers.

\section{Authors' contributions}

$Z Y$ and XC conceived and designed the study. XC performed the experiments. SL, YL and SW collected and analyzed the data. MZ, AZ and JZ contributed materials. XC wrote the paper. ZY edited the paper. All authors read and approved the final manuscript.

\section{Funding}

This study was supported by Project supported by the Natural Science Foundation of Fujian Province, China (Grant Number: 2018J01239.)

\section{Availability of data and materials}

The data and material used to support the findings of this study are available from the corresponding author upon request.

\section{Declarations}

Ethics approval and consent to participate

All of the animal studies were approved by the Institutional Animal Care and Use Committees of Fujian Medical University. The use of papillary thyroid carcinoma tissues was approved by the Ethics Committee of Fujian Medical University Provincial Clinical Medical College, Fujian Provincial Hospital. Informed consent was obtained from all patients.

\section{Consent for publication}

All authors read and approved the final manuscript for publication.

\section{Competing interests}

The authors declare that they have no competing interests.

\section{Author details}

${ }^{1}$ Department of General Surgery, Fujian Medical University Provincial Clinical Medical College, Fujian Provincial Hospital, Fuzhou 350001, Fujian, China.

${ }^{2}$ Fujian Medical University, Fuzhou 350001, Fujian, China. ${ }^{3}$ Department of Paediatric Surgery, Fujian Medical University Provincial Clinical Medical College, Fujian Provincial Hospital, Fujian Medical University, Fuzhou 350000, Fujian, China. ${ }^{4}$ Department of Obstetrics and Gynaecology, The Hospital of Changle District, Fuzhou 350200, China. ${ }^{5}$ Department of Ultrasonography, Fujian Medical University Provincial Clinical Medical College, Fujian Provincial Hospital, Fuzhou 350001, China. ${ }^{6}$ Department of Ultrasound, Fujian Medical University Union Hospital, Fuzhou 350001, Fujian, China.

Received: 4 August 2021 Accepted: 17 January 2022

Published online: 05 February 2022

\section{References}

1. Sun W, Qin Y, Wang Z, Dong W, He L, Zhang T, Zhang H. The NEAT1_2/ miR-491 axis modulates papillary thyroid cancer invasion and metastasis through TGM2/NFkb/FN1 signaling. Front Oncol. 2021;11: 610547.

2. Davies L, Welch HG. Current thyroid cancer trends in the United States. JAMA Otolaryngol Head Neck Surg. 2014;140(4):317-22.

3. Luo Q, Guo F, Fu Q, Sui G. hsa_circ_0001018 promotes papillary thyroid cancer by facilitating cell survival, invasion, $\mathrm{G}(1) / \mathrm{S}$ cell cycle progression, and repressing cell apoptosis via crosstalk with miR-338-3p and SOX4. Mol Ther Nucleic Acids. 2021;24:591-609.

4. Tang B, Zhu J, Li J, Fan K, Gao Y, Cheng S, Kong C, Zheng L, Wu F, Weng $\mathrm{Q}$, et al. The ferroptosis and iron-metabolism signature robustly predicts clinical diagnosis, prognosis and immune microenvironment for hepatocellular carcinoma. Cell Commun Signal. 2020;18(1):174.

5. Li B, Huang Z, Yu W, Liu S, Zhang J, Wang Q, Wu L, Kou F, Yang L. Molecular subtypes based on CNVs related gene signatures identify candidate prognostic biomarkers in lung adenocarcinoma. Neoplasia. 2021;23(7):704-17.

6. Xie Z, Li X, He Y, Wu S, Wang S, Sun J, He Y, Lun Y, Xin S, Zhang J. Analysis of the prognostic value and potential molecular mechanisms of TREM-1 overexpression in papillary thyroid cancer via bioinformatics methods. Front Endocrinol. 2021;12: 646793.

7. Barbaro D, Incensati RM, Materazzi G, Boni G, Grosso M, Panicucci E, Lapi P, Pasquini C, Miccoli P. The BRAF V600E mutation in papillary thyroid cancer with positive or suspected pre-surgical cytological finding is not associated with advanced stages or worse prognosis. Endocrine. 2014;45(3):462-8.

8. Al-Salam S, Sharma C, Afandi B, Al Dahmani K, Al-Zahrani AS, Al Shamsi A, Al Kaabi J. BRAF and KRAS mutations in papillary thyroid carcinoma in the United Arab Emirates. PloS ONE. 2020;15(4): e0231341.

9. Dong $X$, Song J, Hu J, Zheng C, Zhang X, Liu H. T-Box transcription factor 22 is an immune microenvironment-related biomarker associated with the BRAF (V600E) mutation in papillary thyroid carcinoma. Front Cell Dev Biol. 2020;8: 590898. 
10. Park KS, Saindane M, Yang EY, Jin T, Rallabandi HR, Heil A, Nam SE, Yoo YB, Yang JH, Kim JB, et al. Selective inhibition of V600E-mutant BRAF gene induces apoptosis in thyroid carcinoma cell lines. Ann Surg Treat Res. 2021;100(3):127-36.

11. Chen J, Li XL, Zhang YF, Wang D, Wang Q, Zhao CK, Li MX, Wei Q, Ji G, Xu HX. Ultrasound validation of predictive model for central cervical lymph node metastasis in papillary thyroid cancer on BRAF. Future Oncol. 2020;16(22):1607-18.

12. Eozenou C, Gonen N, Touzon MS, Jorgensen A, Yatsenko SA, Fusee L, Kamel AK, Gellen B, Guercio G, Singh P, et al. Testis formation in XX individuals resulting from novel pathogenic variants in Wilms'tumor 1 (WT1) gene. Proc Natl Acad Sci USA. 2020;117(24):13680-8.

13. Wang Y, Weng WJ, Zhou DH, Fang JP, Mishra S, Chai L, Xu LH. Wilms tumor 1 mutations are independent poor prognostic factors in pediatric acute myeloid leukemia. Front Oncol. 2021;11:632094.

14. Hohenstein P, Hastie ND. The many facets of the Wilms'tumour gene, WT1. Hum Mol Genet. 2006;15(2):R196-201.

15. Hastie ND. Wilms'tumour 1 (WT1) in development, homeostasis and disease. Development. 2017;144(16):2862-72.

16. Qi XW, Zhang F, Yang XH, Fan $\sqcup$, Zhang Y, Liang Y, Ren L, Zhong L, Chen $\mathrm{QQ}$, Zhang KY, et al. High Wilms' tumor 1 mRNA expression correlates with basal-like and ERBB2 molecular subtypes and poor prognosis of breast cancer. Oncol Rep. 2012;28(4):1231-6.

17. Lu J, Gu Y, Li Q, Zhong H, Wang X, Zheng Z, Hu W, Wen L. Wilms'tumor 1 (WT1) as a prognosis factor in gynecological cancers: A meta-analysis. Medicine. 2018;97(28): e11485.

18. Bejrananda T, Phukaoloun M, Boonpipattanapong T, Wanitsuwan W, Kanngern S, Sangthong R, Sangkhathat S. WT1 expression as an independent marker of poor prognosis in colorectal cancers. Cancer Biomark. 2010;8(1):35-42.

19. Kim KH, Lee MS. Autophagy — a key player in cellular and body metabolism. Nat Rev Endocrinol. 2014;10(6):322-37.

20. Choi AM, Ryter SW, Levine B. Autophagy in human health and disease. N Engl J Med. 2013;368(7):651-62.

21. Elmore S. Apoptosis: a review of programmed cell death. Toxicol Pathol. 2007;35(4):495-516.

22. Mo H, He J, Yuan Z, Mo L, Wu Z, Lin X, Liu B, Guan J. WT1 is involved in the Akt-JNK pathway dependent autophagy through directly regulating Gas 1 expression in human osteosarcoma cells. Biochem Biophys Res Commun. 2016:478(1):74-80.

23. Zhang Y, Yan WT, Yang ZY, Li YL, Tan XN, Jiang J, Zhang Y, Qi XW. The role of WT1 in breast cancer: clinical implications, biological effects and molecular mechanism. Int J Biol Sci. 2020;16(8):1474-80.

24. Yang J, Zhou Y, Xie S, Wang J, Li Z, Chen L, Mao M, Chen C, Huang A, Chen $Y$, et al. Metformin induces ferroptosis by inhibiting UFMylation of SLC7A11 in breast cancer. J Exp Clin Cancer Res. 2021;40(1):206.

25. Tang B, Zhu J, Fang S, Wang Y, Vinothkumar R, Li M, Weng Q, Zheng L, Yang Y, Qiu R, et al. Pharmacological inhibition of MELK restricts ferroptosis and the inflammatory response in colitis and colitis-propelled carcinogenesis. Free Radical Biol Med. 2021;172:312-29.

26. Zhang M, Weng W, Zhang Q, Wu Y, Ni S, Tan C, Xu M, Sun H, Liu C, Wei P, et al. The IncRNA NEAT1 activates Wnt/ $\beta$-catenin signaling and promotes colorectal cancer progression via interacting with DDX5. J Hematol Oncol. 2018;11(1):113.

27. Macerola E, Proietti A, Poma AM, Ugolini C, Torregrossa L, Vignali P, Basolo A, Materazzi G, Elisei R, Santini F, et al. Molecular alterations in relation to histopathological characteristics in a large series of pediatric papillary thyroid carcinoma from a single institution. Cancers. 2021. https://doi. org/10.3390/cancers13133123.

28. La Vecchia C, Malvezzi M, Bosetti C, Garavello W, Bertuccio P, Levi F, Negri E. Thyroid cancer mortality and incidence: a global overview. Int J Cancer. 2015;136(9):2187-95.

29. Lin X, Wang ZY, Xue G, Qin XJ, Wu JF, Zhang G. ADORA1 is a diagnosticrelated biomarker and correlated with immune infiltrates in papillary thyroid carcinoma. J Cancer. 2021;12(13):3997-4010.

30. Han Y, Yu X, Yin Y, Lv Z, Jia C, Liao Y, Sun H, Liu T, Cong L, Fei Z, et al. Identification of potential BRAF inhibitor joint therapy targets in PTC based on WGCAN and DCGA. J Cancer. 2021;12(6):1779-91.

31. Mancikova V, Buj R, Castelblanco E, Inglada-Pérez L, Diez A, de Cubas AA, Curras-Freixes M, Maravall FX, Mauricio D, Matias-Guiu X, et al. DNA methylation profiling of well-differentiated thyroid cancer uncovers markers of recurrence free survival. Int J Cancer. 2014;135(3):598-610.

32. Oji Y, Miyoshi Y, Koga S, Nakano Y, Ando A, Nakatsuka S, Ikeba A, Takahashi E, Sakaguchi N, Yokota A, et al. Overexpression of the Wilms'tumor gene WT1 in primary thyroid cancer. Cancer Sci. 2003;94(7):606-11.

33. White E, Lattime EC, Guo JY. Autophagy regulates stress responses, metabolism, and anticancer immunity. Trends Cancer. 2021;7(8):778-89.

34. Qiao L, Wong BC. Targeting apoptosis as an approach for gastrointestinal cancer therapy. Drug Resist Updat. 2009;12(3):55-64.

35. Han Z, Liu D, Chen L, He Y, Tian X, Qi L, Chen L, Luo Y, Chen Z, Hu X, et al. PNO1 regulates autophagy and apoptosis of hepatocellular carcinoma via the MAPK signaling pathway. Cell Death Dis. 2021;12(6):552.

36. Carneiro BA, El-Deiry WS. Targeting apoptosis in cancer therapy. Nat Rev Clin Oncol. 2020;17(7):395-417.

37. Aoki M, Fujishita T. Oncogenic roles of the PI3K/AKT/mTOR axis. Curr Top Microbiol Immunol. 2017:407:153-89.

38. Alzahrani AS. PI3K/Akt/mTOR inhibitors in cancer: at the bench and bedside. Semin Cancer Biol. 2019:59:125-32.

39. Tsai JJ, Chen JH, Chen CH, Chung JG, Hsu FT. Apoptosis induction and ERK/NF-KB inactivation are associated with magnolol-inhibited tumor progression in hepatocellular carcinoma in vivo. Environ Toxicol. 2020;35(2):167-75.

40. Yang HL, Liu HW, Shrestha S, Thiyagarajan V, Huang HC, Hseu YC. Antrodia salmonea induces apoptosis and enhances cytoprotective autophagy in colon cancer cells. Aging. 2021;13(12):15964-89.

41. Zeng C, Zou T, Qu J, Chen X, Zhang S, Lin Z. Cyclovirobuxine D inducedmitophagy through the p65/BNIP3/LC3 axis potentiates its apoptosisinducing effects in lung cancer cells. Int J Mol Sci. 2021. https://doi.org/ 10.3390/ijms22115820

42. Han S, Li X, Liang X, Zhou L. HOXA9 transcriptionally promotes apoptosis and represses autophagy by targeting NF-KB in cutaneous squamous cell carcinoma. Cells. 2019. https://doi.org/10.3390/cells8111360.

43. Buffet $C$, Hecale-Perlemoine K, Bricaire L, Dumont F, Baudry C, Tissier F, Bertherat J, Cochand-Priollet B, Raffin-Sanson ML, Cormier F, et al. DUSP5 and DUSP6, two ERK specific phosphatases, are markers of a higher MAPK signaling activation in BRAF mutated thyroid cancers. PlOS ONE. 2017;12(9): e0184861.

44. Qi T, Rong X, Feng Q, Sun H, Cao H, Yang Y, Feng H, Zhu L, Wang L, Du Q. Somatic mutation profiling of papillary thyroid carcinomas by wholeexome sequencing and its relationship with clinical characteristics. Int J Med Sci. 2021;18(12):2532-44.

\section{Publisher's Note}

Springer Nature remains neutral with regard to jurisdictional claims in published maps and institutional affiliations.

Ready to submit your research? Choose BMC and benefit from:

- fast, convenient online submission

- thorough peer review by experienced researchers in your field

- rapid publication on acceptance

- support for research data, including large and complex data types

- gold Open Access which fosters wider collaboration and increased citations

- maximum visibility for your research: over $100 \mathrm{M}$ website views per year

At BMC, research is always in progress.

Learn more biomedcentral.com/submissions 